\title{
Effect of Aerobic Exercises in Prevention of Bone Mass Loss Post Bariatric Surgeries Hassan Mohammed Elkholy ${ }^{1}$, Nessrien Afify Abd Elrashid ${ }^{1}$, Asem Fayed Moustafa ${ }^{2}$, Ahmed Mohamed Nagy Saleh ${ }^{1}$ \\ Departments of ${ }^{1}$ Physical Therapy for Surgery and ${ }^{2}$ General Surgery, Faculty of Physical Therapy, Cairo University, Giza, Egypt *Corresponding author: Hassan Mohammed Elkholy, Mobile: (+20) 01006625259, E-Mail: hassanelkholy921@ gmail.com
}

\begin{abstract}
Background: Patients who have been submitted to bariatric surgery are prone to bone mass abnormalities caused by restriction of calcium intake associated with malabsorption of both calcium and vitamin D. Reduced calcium absorption is secondary to the exclusion of duodenum and proximal jejunum where calcium is maximally absorbed. Aerobic weight bearing exercise is more adapted to improve bone mass over whatever viable physical activity, they assume a double part over stressing the bones as they set both gravitational and muscular stress on bones.

Objective: To determine the effect of aerobic exercises in prevention of bone mass loss post bariatric surgeries.

Patients and Methods: Thirty female patients with post bariatric surgeries participated in this, their ages were between 25 to 45 years selected from Shebin El-Kom Hospital, Menoufia and randomly distributed into two equal groups: Group A (Study group): included 15 patients who received 30 minutes of aerobic exercise through walking on treadmill, in addition to conventional medical care 3 days per week and group B (Control group): included 15 patients who were on their conventional medical care.

Results: There was no significant difference in T score of spine (L2-L4), femoral neck and total femur between both groups pre-treatment $(\mathrm{p}>0.05)$. Comparison between both groups post treatment revealed a significant increase in $\mathrm{T}$ score of spine (L2-L4), femoral neck and total femur of the study group compared with that of the control group $(\mathrm{p}<0.001)$. Conclusion: It can be concluded that aerobic exercises had fruitful effects in cases of osteoporosis patients after bariatric surgery as evidenced by the significant increase in BMD and T-score mean, so decrease risk of fractures and enhance physical performance.
\end{abstract}

Keywords: Bariatric Surgeries, Bone density, DEXA, Obesity.

\section{INTRODUCTION}

Bariatric surgery has become an increasingly common treatment for severe obesity, as it results in significant, sustained weight loss, reverses many complications of obesity and decreases mortality ${ }^{(1)}$. It includes a variety of procedures performed on people who have obesity, weight loss is achieved by reducing the size of the stomach with a gastric band or through removal of a portion of the stomach (sleeve gastrectomy or biliopancreatic diversion with duodenal switch) or by resecting and re-routing the small intestine to a small stomach pouch (gastric bypass surgery) ${ }^{(2)}$. However, these procedures have beneficial effects on many cardiometabolic outcomes, they have negative effects on bone homeostasis, mineral metabolism, including vitamin $\mathrm{D}$ deficiency, hyperparathyroidism and bone loss that persist for at least several years ${ }^{(\mathbf{3})}$.

The most consistent site for bone loss after all bariatric procedures is at the hip ${ }^{(4)}$. Exercise is generally considered to be a safe and efficacious approach to restoring physiological function in patients post-surgery (5). Exercise influenced the skeleton by three main mechanisms; a direct impact on bone that is translated into biological signals by mechanoreceptors , an indirect impact by inducing changes in hormone levels and local factors $^{(\mathbf{6})}$. Aerobic weight bearing exercise is more adapted to improve bone mass over whatever viable physical activity, they assume a double part over stressing the bones as they set both gravitational and muscular stress on bones ${ }^{(5)}$. One of the most well-known types of aerobic exercise is walking, an exercise delicately very well and accepted by the older, as it is harmless, self-managed and effortlessly practicable. The impacts of walking on BMD have been widely considered ${ }^{(7)}$. Treadmill exercise stimulates bone formation and suppresses bone resorption, increases the serum 1,25-dihydroxyvitamin D3 level, and decreases the serum parathyroid hormone level, resulting in an increase in bone mass with stimulation of longitudinal bone growth, especially at weight-bearing sites ${ }^{(\mathbf{8})}$.

The aim of this work was to evaluate the therapeutic effect of aerobic exercises in reducing bone density loss post bariatric surgeries.

\section{PATIENTS AND METHODS}

Thirty female patients with post bariatric surgeries participated in this study. Their ages were between 25 to 45 years selected from Shebin El-Kom Hospital, Menoufia and were randomly distributed into two equal groups:

Group A (Study group): included 15 patients who received 30 minutes of aerobic exercise through walking on treadmill, in addition to conventional medical care (vitamin D supplements and calcium) 3 days /week.

Group B (Control group): includes 15 patients who were only on their conventional medical care (vitamin D supplements and calcium).

Ethical approval:

Research Ethics Committee and quality control approvals were obtained from Cairo University. The 
study purpose and procedures were explained in details and in plain terms to each of the subjects before being asked to give an informed written consent to participate in the study.

\section{Criteria for the patient selection:}

a- Inclusion Criteria:

- Age ranged between 25 to 45 years.

- All patients have bariatric surgery mainly bypass $\mathbf{C}$. surgery

- All patients have no bone diseases.

- All patients were medically stable for 3 months.

- All patients enrolled to the study had signed consent form.

- All the patients examined medically by physician.

b- Exclusion Criteria:

- Bone diseases.

- Any medical condition that affect bone health

- Female patient who have post-menopausal osteoporosis.

- Cardiac diseases.

- Pulmonary diseases.

- Unstable head and neck injury.

- Dementia

- Physical/mental incapacity to perform study requirements.

- Chronic kidney disease.

- Hypothyroidism.

\section{Procedures of the study:}

The procedure of this study was divided into two main procedures:

(A) Measurement procedures:

1- Initial Evaluation Procedures: Each subject was examined medically in order to exclude any abnormal medical problems, which previously mentioned.

2-Estimation of Bone mineral density (BMD): DEXA is most often performed on the lower spine and hips. In children and some adults, the whole body is sometimes scanned (9). T-scores was calculated by taking the difference between patients measured BMD and the mean BMD in healthy young adults matched for gender and ethnic groups and expressing the difference relative to the young adult population standard deviation ${ }^{(10)}$.

Measurements was taken as following: Before starting any protocol (pretreatment) and after three months (posttreatment).

(B) Therapeutic procedures:

Electronic Treadmill: (Steelflex XT3300; Korea)

During the three months intervention period, aerobic training program included 20 - 30 minute walking session three times a week at intensity of $60-80 \%$ of target heart rate.

These exercises were divided into 3 phases as follow:

A. Warm up phase: Simple stretching exercises for all muscles groups then walking for 5 minutes on the treadmill at lowest speed (5 kilometer/hour and at 2 degrees angle of inclination).
B. Active phase: Then gradually increased at 2 minute intervals until it reached the initial intensity of 50- 55\% of the maximal heart rate (MHR) in the first 2 weeks, then increased gradually until reaching 55-60\% of MHR in the second 2 weeks, $60-65 \%$ in the third 2 weeks and $65-75 \%$ in the forth 2 weeks, then increase till reach $80 \%$ of MHR by the end of the last 2 weeks.

C. Cool down phase: by walking for 5 minutes on the treadmill at lowest speed (5 kilometer/hour and at 2 degrees angle of inclination) ${ }^{(11)}$.

The patients were taught to stop the exercise and to notify if they felt any dizziness, headache, palpitations, nausea, anxiety, exhaustion or any other adverse effects. The patients' vital signs were examined at least once during exercise $^{(12)}$.

\section{Statistical analysis}

Data were represented as means \pm standard deviation (SD). Normal distribution of data was checked using the Shapiro-Wilk test for all variables. Levene's test for homogeneity of variances was conducted to test the homogeneity between groups. Unpaired t-test was conducted to compare the mean values of age, BMD of spine, femoral neck and total femur between the study and control groups. Paired t-test was conducted for comparison between pre and post treatment in each group. The level of significance for all statistical tests was set at $p<0.05$. All statistical analysis was conducted through the statistical package for the social sciences (SPSS) version 25 for windows (IBM SPSS, Chicago, IL, USA) ${ }^{(13)}$.

\section{RESULTS}

Subject characteristics: The mean \pm SD age of the study group $36.4 \pm 6.27$ years, with maximum value of 45 years and minimum value of 27 years. The mean \pm SD age of the control group was $35.13 \pm 6.42$ years, with maximum value of 45 years and minimum value of 27 years. There was no significance difference between both groups in the mean age values $(\mathrm{p}=0.59)$.

Effect of treatment on $T$ score of spine (L2-L4), femoral neck and total femur:

Within group comparison: There was no significant difference in T score of spine (L2-L4), femoral neck and total femur between pre and post treatment in the study group, while there was a significant decrease in $\mathrm{T}$ score of spine (L2-L4), femoral neck and total femur of the control group post treatment compared with that of pretreatment (Table 1 and figure 1).

Between groups comparison: There was no significant difference in T score of spine (L2-L4), femoral neck and total femur between both groups pre-treatment. Comparison between both groups post treatment revealed a significant increase in $\mathrm{T}$ score of spine (L2L4), femoral neck and total femur of the study group compared with that of the control group (Table 1 and figure 1). 
Table (1): Mean T score of spine (L2-L4), femoral neck and total femur pre and post treatment of the study and control group

\begin{tabular}{|c|c|c|c|c|c|}
\hline T score & $\begin{array}{c}\begin{array}{c}\text { Study group } \\
\mathbf{N}=\mathbf{1 5}\end{array} \\
\overline{\mathrm{X}}+\mathbf{S D}\end{array}$ & $\begin{array}{c}\text { ontrol group } \mathbf{N}=\mathbf{1 5} \\
\bar{x}+\text { SD }\end{array}$ & MD & t- value & p value \\
\hline \multicolumn{6}{|l|}{ Spine } \\
\hline Pre treatment & $0.66 \pm 0.21$ & $0.72 \pm 0.16$ & -0.06 & -0.84 & 0.4 \\
\hline Post treatment & $0.64 \pm 0.19$ & $-1.91 \pm 0.49$ & 2.55 & 18.6 & $0.001 *$ \\
\hline MD & 0.02 & 2.63 & & & \\
\hline$\%$ of change & 3.03 & 365.27 & & & \\
\hline \multirow[t]{2}{*}{ t- value } & 0.5 & 18.72 & & & \\
\hline & $p=0.62$ & $p=0.001 *$ & & & \\
\hline \multicolumn{6}{|l|}{ Femoral neck } \\
\hline Pre treatment & $0.58 \pm 0.19$ & $0.61 \pm 0.17$ & -0.03 & -0.49 & 0.62 \\
\hline Post treatment & $0.62 \pm 0.17$ & $-1.31 \pm 0.81$ & 1.93 & 9.02 & $0.001 *$ \\
\hline MD & -0.04 & 1.92 & & & \\
\hline$\%$ of change & 6.9 & 314.75 & & & \\
\hline \multirow[t]{2}{*}{ t- value } & -1.7 & 8.92 & & & \\
\hline & $p=0.11$ & $p=0.001 *$ & & & \\
\hline \multicolumn{6}{|l|}{ Total femur } \\
\hline Pre treatment & $0.82 \pm 0.29$ & $0.84 \pm 0.17$ & -0.02 & -0.15 & 0.88 \\
\hline Post treatment & $0.86 \pm 0.24$ & $-1.38 \pm 0.78$ & 2.24 & 10.61 & $0.001 *$ \\
\hline MD & -0.04 & 2.22 & & & \\
\hline$\%$ of change & 4.78 & 264.28 & & & \\
\hline \multirow[t]{2}{*}{ t- value } & -1.16 & 11.12 & & & \\
\hline & $p=0.26$ & $p=0.001 *$ & & & \\
\hline
\end{tabular}

$\overline{\mathrm{x}}$, mean; SD, standard deviation; MD, mean difference; $\mathrm{p}$-value, probability value; *, significant

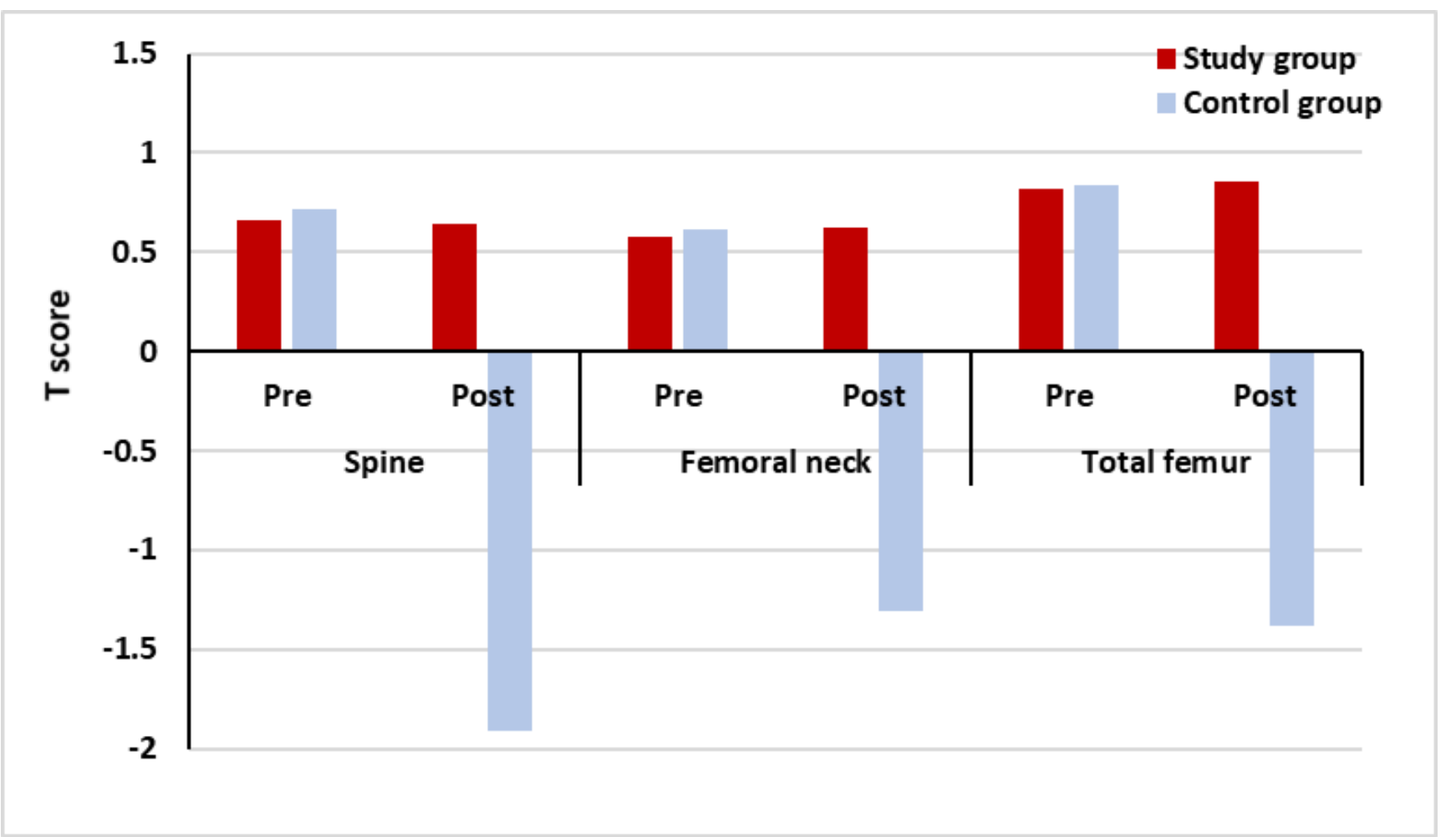

Figure (1): Mean T score of spine (L2-L4), femoral neck and total femur pre and post treatment of the study and control group 


\section{DISCUSSION}

Patients who have been submitted to bariatric surgery are prone to bone mass abnormalities caused by restriction of calcium intake associated with malabsorption of both calcium and vitamin D. Reduced calcium absorption is secondary to the exclusion of duodenum and proximal jejunum where calcium is maximally absorbed. Vitamin $\mathrm{D}$ is absorbed preferentially in the jejunum and ileum ${ }^{(14)}$.

The defective absorption of fat and fat-soluble vitamins including vitamin $\mathrm{D}$ aggravates calcium malabsorption. The relative lack of calcium stimulates the production of parathyroid hormone that in turn increases production of 1,25-dihydroxy vitamin D and increases release of calcium from bone. The result of this process is the long-term risk of osteoporosis ${ }^{(15)}$.

Results of this study are confirmed by study of Blanchet et al. ${ }^{\left({ }^{(6)}\right)}$ and Sievanen ${ }^{\left({ }^{(6)}\right.}$ that exercise has an impact on bone turnover changes that tend towards bone formation in response to exercise of moderate intensity and towards bone resorption with intensive training. Also, Kohrt et al. ${ }^{(17)}$, Welch and Weaver ${ }^{(18)}$ and Robling ${ }^{(19)}$ proved that there is a relationship between physical loads and bone structure and bone responds maximally to mechanical load and it appeared that moderate intensity aerobic training that improves strength and muscle mass also improves bone mass. $70 \%$ of the tension exerted on bone depends on muscle contraction which was therefore more important than body weight itself as a mechanical stimulus. Moreover, Wolff $\boldsymbol{e t}$ al. ${ }^{(20)}$ proved that moderate intensity aerobic exercise three times weekly for three months was associated with improved values of bone mineral density.

Valderas et al. ${ }^{(21)}$ stated that aerobic exercises are considered a promising intervention for reversing the deterioration of muscle structure as well as osteoporotic effects after Roux-en-Y gastric bypass. Mauney et al. ${ }^{(22)}$, Kouidi et al. ${ }^{(23)}$ and Ling et al. ${ }^{(24)}$ found that exercise with sufficient bone loading force, such as aerobic exercise, has been shown to preserve and also increase bone mass and walking is weight bearing activity that has its impact on bone and bone marrow and patients should be encouraged to begin a walking program starting with 10 to $30 \mathrm{~min} /$ day, 3 days/week at a moderate difficulty level as tolerated, then be encouraged to increase their walking time to at least $30 \mathrm{~min}$ on 3 days/week or more, keeping the intensity at a moderate level (or a perceived exertion of "somewhat hard"). Also, Dimeo et al. ${ }^{(5)}$ and Iwamoto et al. ${ }^{(8)}$ have found that weight bearing exercise in forming of walking on treadmill at intensity of $80 \%$ of maximal heart rate is more adapted to improve bone mass than any other physical activity as it stimulates bone formation and suppresses bone resorption, increases the serum 1,25-dihydroxyvitamin D3 level, and decreases the serum parathyroid hormone level, resulting in an increase in bone mass with stimulation of longitudinal bone growth, especially at weightbearing sites.

Moreover, the finding of the present study is consistent with the works reported by Hind $\boldsymbol{e t}$ al. ${ }^{(25)}$ and Douchi et al. ${ }^{(26)}$ that regular aerobic weight bearing exercise sessions, two to three times weekly, over a period of at least 6 months recognized as a major and effective prophylaxis against osteoporosis, to firstly stimulate bone accretion during growth; secondly, to stimulate bone accretion once bone loss has occurred and thirdly, to prevent bone loss. Also, the finding of our study are supported by the finding of Siwapituk and Kitisomprayoonkul ${ }^{(27)}$, that regular walks on a treadmill three times a week for three months, at an intensity of $55-75 \%$ of maximal heart rate, bone turnover rate was determined by measurements taken before the experiment, at one week, and again after the three-month program. The measurements determined levels of crosslinked C-terminal telopeptides of type 1 collagen and $\mathrm{N}$-terminal pro-peptides of type 1 procollagen, both of which are biomarkers of bone formation.

However, results from Howe et al. ${ }^{(28)}$ found that progressive resistance strength training is the most effective on bone density for the neck of femur while the most effective intervention for bone density at the spine has been suggested to be the multicomponent training exercises.

Finally from the results of the present study and the other past review of related literature that treadmill aerobic exercises have positive and scientific effect in improving bone calcium in bariatric patients as evidenced by the statistical results of the present study that showed a statically improvement in the study group; it could concluded that application of the treadmill aerobic exercises:

- Improves bone integrity

- Reduces the great public health burden of osteoporosis patients post bariatric surgery.

- Improves quality of life and functional performance.

\section{CONCLUSION:}

Within the limitations of this study, the following conclusion is warranted:

It can be concluded that aerobic exercises had fruitful effects in cases of osteoporosis in patients after bariatric surgery as evidenced by the significant increase in BMD and T-score mean, so decrease risk of fractures and enhance physical performance.

\section{Future studies and Recommendations:}

1. Further researchers are needed using different aerobic exercise parameters (intensity, frequency of treatment, duration of treatment) to select the best for the patient.

2. Further researches should be carried out using larger sample size (more than 30 patients). 
3. Further researchers are needed to study other physical therapy modalities and its effect on bone mineral density.

4. Greater sample number could be used to assure the results and for a longer period than 3 months.

5. Further studies are needed on different bariatric surgery procedures that affect bone mineral density to open new insights and widen the scope of knowledge about bariatric surgery.

\section{REFERENCES}

1. Mechanick J, Youdim A, Jones D et al. (2013): Clinical practice guidelines for the perioperative nutritional, metabolic, and nonsurgical support of the bariatric surgery patient. official journal of the American Society for Bariatric Surgery, 9(2):159-91

2. Karmali S, Johnson C, Sharma A et al. (2010): Bariatric surgery / Clinical review. Canadian Family Physician, 56: 873-879.

3. Yu E (2014): Bone metabolism after bariatric surgery. Journal of Bone and Mineral Research, 29(7): 1507-1518.

4. Gagnon C, Schafer A (2018): Bone health after bariatric surgery. JBMR Plus, 2(3): 121-133.

5. Dimeo F, Knauf W, Geilhaupt D et al. (2007): Endurance exercise and production of growth hormone and hematopoietic factors in patients with Anemia. British Journal of Sports Medicine, 38 (6): 37-42.

6. Sievanen $\mathbf{H}$ (2005): Hormonal influences on the musclebone feedback system. J Musculoskelet Neuronal Interact, 5(3): 255-61.

7. Kabolam J, van Uffelen Z, Taaffe D (2013): The effect of physical exercise on bone density in middle-aged and older men: a systematic review. Osteoporosis International, 24(11): 2749-2762

8. Iwamoto J, Shimamura C, Takeda T et al. (2004) Effects of treadmill exercise on bone mass, bone metabolism, and calciotropic hormones in young growing rats. J Bone Miner Metab., 22(1):26-31.

9. Binkovitz L, Henwood M (2007): Pediatric DXA: technique and interpretation. Pediatr Radiol., 37 (1): 2131.

10. Yamada M, Ito M, Hayashi K et al. (1994): Dual energy $\mathrm{X}$-ray absorptiometry of the calcaneus: comparison with other techniques to assess bone density and value in predicting risk of spine fracture. AJR Am J Roentgenol., 163:1435-40.

11. Jamtvedt G (2010): A pragmatic randomised trial of stretching before and after physical activity to prevent injury and soreness . British Journal of Sports Medicine, 44:1002-06.

12. Khalighfard S, Gaeini A, Nazarali P (2011): Effect of endurance exercise on cardiac marker and exerciseinduced immune response. Trauma Mon., (1):45-51.
13. Maronna R, Martin R, Yohai V (2006): Robust statistics theory and methods. Journal of the American Statistical Association, 90: 330-341.

14. Heaney R (2004): Functional indices of vitamin D status and remifications of vit D defrciency. Am J Clin Nutr., 80: 1706-1709.

15. Mahdy T, Atia S, Farid M (2008): Effect of Roux-en Y gastric bypass on bone metabolism in patients with morbid obesity Obes Surg., 18: 1526-1531.

16. Blanchet C, Chaire L, Thibault G (2008): Activite physique et sante osseuse. Avis du comite scientifique de Kino-Quebec, 14(1): 1-42.

17. Kohrt W, Bloomfield S, Nelson M et al. (2004): Effects of exercise involving predomintaly either joint reaction or ground reactions forces on bone mineral density in adults. J Bone Miner Res., 13: 353-364.

18. Welch J, Weaver C (2005): Calcium and exercise affect the growing skeleton Nutr Ren., 63: 361-373.

19. Robling A (2009): Is bone response to mechanical signals dominated by muscle forces? Med Sci., 41: 2044-9.

20. Wolff I, Van C, Twis H (2004): The effect of exercise training programs on bone mass Obes Surg., 29: 131-148.

21. Valderas J, Velasco S, Solari S (2009): Increase of bone resorption and the parathyroid hormone in postmenoposal women in the long term after gastric bypass. Obes Surg., 19: 1132-1138.

22. Mauney J, Sjostorm S, Blumberg J et al. (2004): Mechanical stimulation promotes osteogenic differentiation of human bone marrow stromal cells. Calcified Tissue international, 47: 458-468.

23. Kouidi E, Grekas D, Deligiannis A (2009): Effects of exercise training on non-invasive cardiac measures in patients with ESRD : A randomized controlled trial. Am J Kidney Dis., 57: 511-21.

24. Ling L, Lo M, Yao W et al. (2013): The effects of different weight-bearing exercise training on bone mineral density and bone metabolism in young men. Journal of Science and Medicine in Sport, 12(2):123-124.

25. Hind K, Truscott J, Conway S (2008): Exercise during childhood and adolescence. J Cyst Fibrosis, 7(4):270-276.

26. Douchi T, Yamamoto S, Oki T et al. (2010): The effects of physical exercise on body fat distribution and bone mineral density in postmenopausal women. Maturitas, 35(1):25-30.

27. Siwapituk W, Kitisomprayoonkul W (2016): Bone turnover increases during supervised treadmill walking in Thai postmenopausal women. Osteoporosis and Sarcopenia, 2(1) 41-44.

28. Howe T, Shea B, Dawson L et al. (2011): Exercise for preventing and treating osteoporosis in postmenopausal women. Cochrane Database of Systematic Reviews, 1(7):1-167 